\title{
Investigating the Effect of Hydrogen-Rich Water on Liver Cell Injury and Liver Cancer by Regulating GP73/ TGF- $\beta$ Pathway
}

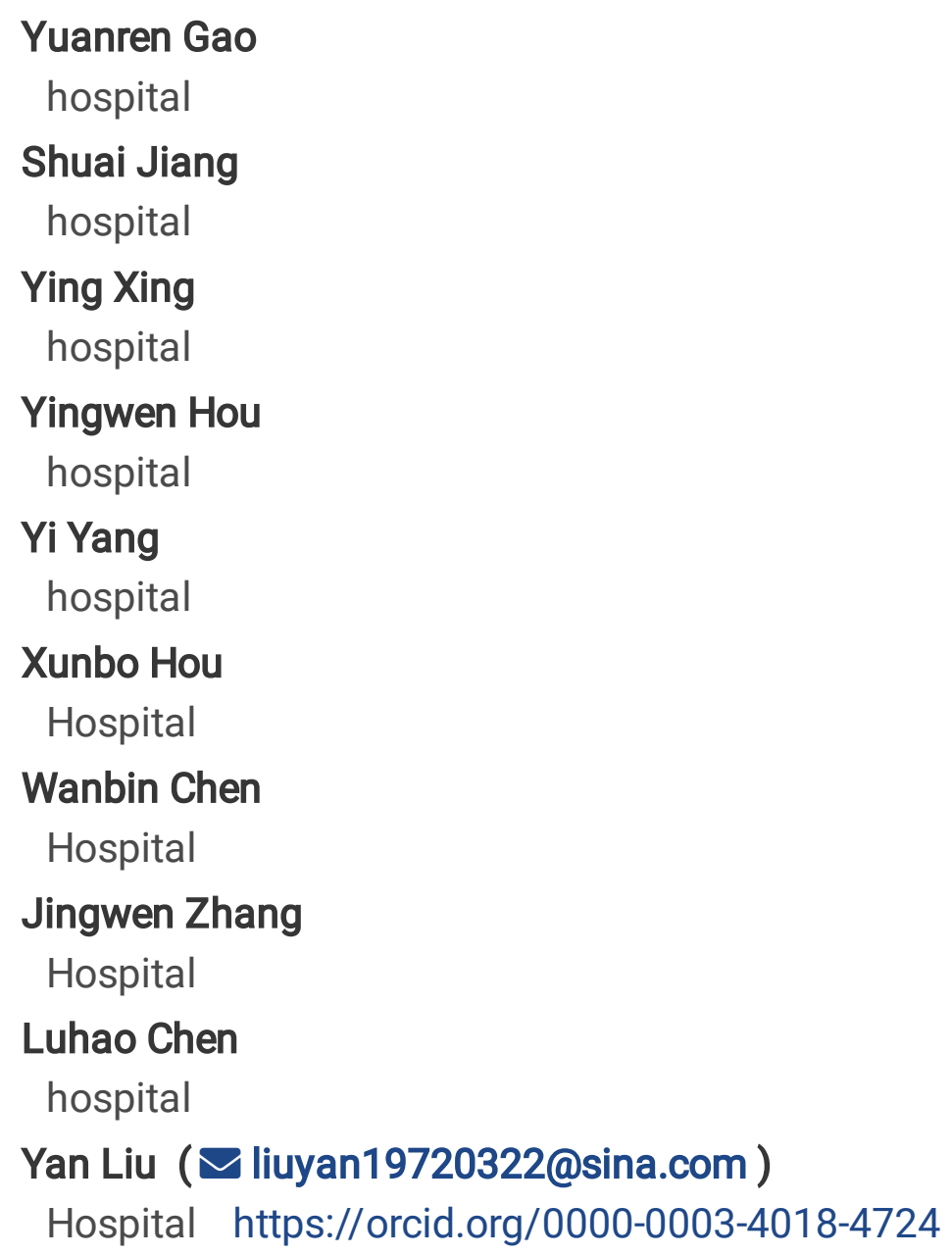

Primary research

Keywords: hydrogen-rich water, liver cancer, transforming growth factor (TGF)- $\beta$, Smad, GP73

Posted Date: February 16th, 2021

DOI: https://doi.org/10.21203/rs.3.rs-201468/v1

License: (1) (1) This work is licensed under a Creative Commons Attribution 4.0 International License. Read Full License 


\section{Abstract}

\section{Background}

Liver cancer is an extremely heterogeneous malignant disease among tumors identified to date. In recent years, a large number of studies have found that low-concentration hydrogen or hydrogen-rich water or hydrogen-saturated physiological saline has a protective effect on many diseases. Observing the intervention effect of hydrogen-rich water and speculate whether its effect is regulated by GP73/TGF- $\beta$ signaling pathway would provide a basis for clinical treatment using hydrogen in liver cancer.

Method

The(N-nitrosodiethylamine) DEN-induced LX-2 hepatocyte injury model, hepatocarcinoma cell(HepG2 cells), and normal hepatic stellate cell LX-2 and HepG2 co-culture system were treated with hydrogen-rich water, and the cell viability was measured by the Cell Counting Kit-8(CCK-8)method. Also, the supernatant was collected to determination the transforming growth factor (TGF- $\beta$ ), tumor necrosis factor (TNF- $\alpha$ ), and osteopontin (OPN) content. Subsequently, qPCR method was employed to detect the expression of GP73, TGF- $\beta$, and Smad2 mRNA in the cell. Total cell protein was extracted, and the level of GP73, TGF- $\beta$, and Smad2 proteins in the cells was detected by Western blot.

Result

After the intervention of hydrogen-rich water, DEN increases the activity of LX-2 hepatocytes, inhibits the secretion of TGF- $\beta$, downregulate the expression of GP73, TGF- $\beta$, and Smad2 mRNA in the injured LX-2 cells, inhibits the expression of GP73 and TGF- $\beta$ proteins, and exerts a protective effect on injured cells. The intervention with hydrogen-rich water in HepG2 liver cancer cells inhibits the expression of GP73 mRNA and protein in the cells, upregulates the expression of TGF- $\beta$ mRNA and protein, inhibits the expression of OPN, and exerts an anti-proliferation effect on liver cancer cells. In the co-culture system of LX-2 and HepG2, the effect of hydrogen-rich water showed different effects with the altered ratio of the two cells in the system.

Conclusion

Hydrogen-rich water has a protective effect on liver cell damage and an inhibitory effect on liver cancer cells, which is effectuated by regulating the GP73/TGF- $\beta$ signaling pathway.

\section{Introduction}

Liver cancer is an extremely heterogeneous malignant disease among tumors identified to date. Hepatocellular carcinoma (HCC) arises from chronic liver inflammation and fibrosis and takes a varied course in individual patients up to tumor formation. Risk factors, such as hepatitis B virus (HBV) and/or hepatitis C virus (HCV) infections, aflatoxin infection, abused alcohol intake, metabolic syndrome, obesity, and diabetes are closely related to the environmental and genetic susceptibilities to HCC [1]. Golgi protein 
73(GP73) is a type II Golgi membrane protein, which is a transmembrane glycoprotein. Kladney et al. [2] first isolated Golgi protein GP73 during a genetic screening from the liver of a patient with adult giant-cell hepatitis (GCH). The expression of GP73 is increased markedly in HCC cells, and the serum levels are predictive of HCC [3-5]. Several studies have reported GP73 as a serum marker for HCC [6-8]. In patients with acute and chronic hepatitis, cirrhosis, and liver cancer, the level of GP73 is significantly increased. TGF- $\beta$ belongs to the superfamily of transforming growth factor-beta (TGF- $\beta$ ) signaling molecules and is widely expressed across nematodes to mammals. It is synthesized and secreted in an inactive form in all types of cells, and only after activation and binding to the receptor, it exhibits biological activity and pathophysiological effects. Furthermore, TGF- $\beta$ is a multifunctional cytokine that is regulated via the TGF- $\beta /$ Smad 2 signaling pathway and closely related to the occurrence, invasion, and metastasis of many tumors [9]. The TGF- $\beta$ signaling pathway interferes with hepatocyte proliferation, and also accelerates HCC progression. Thus, TGF- $\beta$ has been reported to play both tumor-suppressive and tumor-promoting roles [10].

Hydrogen is a reductive small gaseous molecule that exhibits "antioxidant," "anti-inflammatory," and "anti-apoptotic" effects in vivo [11,12]. In recent years, a large number of studies have found that lowconcentration hydrogen or hydrogen-rich water or hydrogen-saturated physiological saline has a protective effect on many diseases. Some studies have shown that hydrogen-rich water significantly reduces the side effects caused by radiotherapy and improves the quality of life of radiotherapy patients. On the other hand, a few studies have shown that hydrogen-rich water has a certain preventive effect on kidney cancer and liver cancer caused by non-alcoholic steatohepatitis. In this study, we established Nnitrosodiethylamine (DEN)-induced hepatic stellate cell injury model, liver cancer HepG2 cells, and HepG2 and LX-2 co-culture system to observe the intervention effect of hydrogen-rich water and speculate whether its effect is regulated by GP73/TGF- $\beta$ signaling pathway. These findings would provide a basis for clinical treatment using hydrogen in liver cancer.

\section{Materials And Methods}

Human liver cancer HepG2 cells (Beijing Duan-Dian Pharmaceutical Research and Development Co., Ltd, Beijing, China), human stellate liver cell LX-2 (Zeye Bio), DEN (Sigma, Missouri, U.S. Lot\#049K161), Hydrogen-rich water (purchased from Wuxi Yihydroquan Food Co., Ltd, Jiangsu, China), RNA extraction and reverse transcription kit (Novoprotein, Jiangsu, China E047), quantitative PCR kit (Thermo, Massachusetts, USA, 1911596), total protein extraction kit (Solarbio, Beijing, China, BC3710), GP73 antibody (Bioss, Beijing, China, bs-4887R), TGF- $\beta$ antibody (Bioss, Beijing, China, bs-0086R), and Smad2 antibody (SANTA CRUZ, sc-376928) were also procured.

LX-2 cells were cultured in RPMI 1640 (Giboc, New York, USA, NO.C11875500BT) containing 10\% fetal bovine serum (FBS) and various concentrations of DEN (10, 25, 50, 80, 100, 160, and $200 \mu \mathrm{mol} / \mathrm{L}$, respectively) for $16 \mathrm{~h}$. The cell survival was detected by the CCK8. The concentration of LX-2 cells in the logarithmic growth phase was adjusted to $1 \times 104$ cells $/ \mathrm{mL}$, and a volume of $200 \mu \mathrm{L}$ was plated/well in a 96-well culture plate. Subsequently, the cells were divided into normal, DEN model, and test groups 
(including hydrogen-rich water medium group). The normal and DEN model groups did not receive any treatment. The test group was treated with hydrogen-rich water for $16 \mathrm{~h}$, and the cell survival rate was detected by the Cell Counting Kit-8 (CCK-8) method to observe the protective effect of hydrogen-rich water on LX-2 cells. HepG2 cells were cultured with RPMI 1640 containing $10 \%$ FBS, the cell density was adjusted to $1 \times 104$ cells $/ \mathrm{mL}$, and $200 \mu \mathrm{L} /$ well dispensed in a 96-well culture plate. Then, 1, 2, 3, and 4 number of times hydrogen-rich water and ionized water medium were added for $8 \mathrm{~h}$ and $24 \mathrm{~h}$, respectively, following which, the cell viability was detected by CCK-8 assay. The LX-2 and HepG2 cells were co-cultured at the ratios of 1:3,1:1, and 3:1, respectively. Hydrogen-rich water was added to the coculture system for $24 \mathrm{~h}$, and the cell viability was detected by the CCK-8 method. After the intervention of the above-mentioned cells and hydrogen-rich water, the cell supernatant was collected, and the content of transforming growth factor (TGF- $\beta$ ), tumor necrosis factor-alpha (TNF-a), and osteopontin (OPN) was detected by enzyme-linked immunosorbent assay (ELISA). The above-mentioned cell culture and hydrogen-rich water intervention groups were subjected to total cell RNA extraction and reverse transcription. Real-time PCR was used to quantify GP73, TGF- $\beta$, and Smad 2 mRNA, and $\beta$-actin was used as an internal reference to calculate the relative expression of the target genes using the $2^{-\triangle \triangle C T}$ method.

\section{Table 1. Primer sequences}

\begin{tabular}{|ll|}
\hline Gene & Sequence ( ${ }^{\prime}$ 'to 3' $^{\prime}$ ) \\
\hline GP73-F & AGAGCGAGGTGCCGTAGAGC \\
\hline GP73-R & GCCTTCTCGTCCTGGTGTAACTTG \\
\hline TGF- $\beta$-F & CGGCAGCTGTACATTGACTT \\
\hline TGF- $\beta$-R & TCAGCTGCACTTGCAGGAGC \\
\hline Smad2-F & ACTATACCCACTCCATTCCA \\
\hline Smad2-R & CACTATCACTTAGGCACTCG \\
\hline$\beta$-actin-F & CCTCACTGTCCACCTTCCA \\
\hline$\beta$-actin-R & GGGTGTAAAACGCAGCTCA \\
\hline
\end{tabular}

Total protein was extracted from the above cell culture and hydrogen-rich water intervention groups and quantified. An equivalent of $20 \mu \mathrm{g}$ total protein was separated by sodium dodecyl sulfate polyacrylamide gel electrophoresis (SDS-PAGE), transferred to PVD membranes (Millipore, Beijing, China, NO.R8HA7228), blocked, and probed with primary antibodies overnight at $4{ }^{\circ} \mathrm{C}$. Subsequently, the membranes were incubated with the corresponding secondary antibodies at room temperature. Finally, the immunoreactive bands were visualized using enhanced chemiluminescence (ECL) and images acquired.

All data were analyzed using SPSS16.0 software and expressed as $x \pm S D$. One-way analysis of variance (ANOVA) was used for comparison among multiple groups, and $\mathrm{P}<0.05$ indicated statistical significance. The plots were constructed using GraphPad Prism 8. 


\section{Results}

\subsection{The protective effect of hydrogen-rich water on the damage of $L X-2$ cells induced by DEN}

Different concentrations of DEN were used to intervene in LX-2 cells. As the concentration of DEN increased, the cell activity of LX-2 decreased, and the content of TGF- $\beta$ and TNF- $\alpha$ in the cell supernatant showed an increasing trend. When the concentration of DEN is $200 \mu \mathrm{mol} / \mathrm{L}$, the cell activity of LX-2 is significantly reduced compared to the control group without DEN (Fig. 1A). Also, the contents of TGF- $\beta$ and TNF- $\alpha$ in the supernatant increased (Fig. 1B and C). In the presence of different concentrations of DEN in LX-2 cells, the medium prepared with hydrogen-rich water was replaced in the experimental group. These results showed that when the concentration of DEN was $200 \mu \mathrm{mol} / \mathrm{L}$, the cell viability of the experimental group was increased significantly compared to the model group without hydrogen-rich water $(P<0.001)$ (Fig. 1D). Compared with the model group, the content of TGF- $\beta$ in the supernatant of the experimental group increased slightly (Fig. 1E), and the content of TNF-a increased significantly (Fig. 1F). Therefore, DEN causes damage to LX-2, and after hydrogen-rich water intervention, it exerts a protective effect on DEN-induced cell damage.

Real-time PCR results showed that when $200 \mu \mathrm{mol} / \mathrm{L}$ DEN was used to induce LX-2 cells, the expression of GP73, TGF- $\beta$, and Smad2 mRNA was upregulated to varying degrees compared to normal LX-2 cells (Fig. 2A-C). After changing to a hydrogen-rich medium, GP73, TGF- $\beta$, and Smad 2 mRNA in the cells was significantly downregulated compared to the model group (Fig. 2A-C). Western blot results showed that after hydrogen-rich water interfered with DEN-induced LX-2 cells, the protein level of GP73 and TGF- $\beta$ in the cells was decreased compared to the model group, but no significant inhibitory effect was noted on the level of Smad2 protein (Fig. 2D-G). This phenomenon showed that hydrogen-rich water protects DEN from damage by inhibiting the expression of GP73 and TGF- $\beta$ mRNA and protein in the cells. It is also speculated that DEN induced damage in LX-2 cells, but the damage did not progress to the stage of liver cancer, and hence, hydrogen-rich water did not have a significant effect on the protein expression of Smad2 in the cells.

\subsection{The effect of hydrogen-rich water on HepG2 liver cancer cells}

The cell viability data of HepG2 cells treated with hydrogen-rich water culture medium for $8 \mathrm{~h}$ showed that the viability of HepG2 cells with three volumes of hydrogen-rich medium decreased significantly, indicating an inhibitory effect of hydrogen on HepG2 cells (Fig. 3A). Similarly, after $24 \mathrm{~h}$ of HepG2 cell culture medium prepared with hydrogen-enriched water for different durations, the activity of the cells given hydrogen-enriched medium three times decreased significantly compared to the control group without hydrogen-enriched water medium $(P<0.05)$ (Fig. 3B). HepG2 intervened with hydrogen-rich medium three times and treated for $24 \mathrm{~h}$ showed that when the number of HepG2 cells was 5000 cells/well, hydrogen gas significantly inhibited the activity of liver cancer cells as compared to the control group without hydrogen-enriched water $(\mathrm{P}<0.01)$, and a maximum inhibitory effect was also observed (Fig. 3C). The determination of TGF- $\beta$ and TNF- $\alpha$ in the cell supernatant showed increased secretion of TGF- $\beta$ after adding hydrogen-rich medium for different times. When the hydrogen-rich medium was given 
three times simultaneously, the level of TNF-a increased in the supernatant of the cells, which in turn inhibited the proliferation of liver cancer cells (Fig. 3D and E). Thus, the addition of hydrogen-rich medium three times was preferred as the optimal conditions to intervene in HepG2 cells. The results showed that hydrogen intervention increases the content of TGF- $\beta$ and TNF- $\alpha$ in the supernatant of HepG 2 cells, which is speculated to elevate TGF- $\beta$-inhibited degradation of extracellular matrix and prevent the migration and invasion of liver cancer (Fig. $3 \mathrm{~F}$ and G).

The intervention of HepG2 cells with a hydrogen-rich medium inhibited the expression of GP73 and Smad 2 mRNA in the cells (Fig. 4A and C) and increased the expression of TGF- $\beta$ mRNA (Fig. 4B). Western blot showed that after intervention with hydrogen-rich medium, the expression of GP73 protein was downregulated in the cells (Fig. 4E), and that of TGF- $\beta$ protein was upregulated (Fig. 4F), but no significant change was detected in Smad2 protein. (Fig. 4G). Thus, it was speculated that hydrogen not only passes through the GP73/TGF- $\beta$ pathway but might also inhibit the proliferation of liver cancer cells via other pathways.

\subsection{Study on the effect of hydrogen-rich water on the co-culture system of LX-2 and HepG2}

The co-cultivating of LX-2 and HepG2 cells at different ratios revealed that the growth rate of LX-2 cells was significantly lower than that of HepG2 cells. When the two types of cells were co-cultured for $4 \mathrm{~h}$, HepG2 cells were found to fuse with LX-2 cells, as assessed by microscopy. With prolonged culture time, the fusion effect became pronounced, indicating that HepG2 cells are highly invasive (Fig. 5).

The hydrogen-rich medium was added to the co-culture system of LX-2 and HepG2 cells (1:1) in different proportions. Microscopic analysis revealed that hydrogen intervention exerts a significant inhibitory effect on cell proliferation (Fig. 6A, 6B). This phenomenon is consistent with the inhibitory effect of the previous hydrogen-rich medium on different numbers of HepG2. When LX-2 cells are dominant in the co-culture system, hydrogen increases the secretion of TGF- $\beta$ in the cell supernatant (Fig. $6 \mathrm{C} 1$ ), thereby promoting the proliferation of normal liver cells in the co-culture system to combat the invasion of normal cells by liver cancer cells. Furthermore, in the co-culture system, with the increase in the number of HepG2 cells in hepatocytes, hydrogen gas inhibits the secretion of TGF- $\beta$ and prevents excessive secretion of TGF- $\beta$ from causing excessive proliferation of liver cancer cells (Fig. 6D1). When the HCC cells are dominant in the co-culture system, although the hydrogen-rich medium does not inhibit the secretion of TGF- $\beta$ after the intervention, the level of TNF-a is elevated (Fig. $6 \mathrm{C} 2$, D2, and E2), and the inflammatory factors kill the tumor cells. In order to further explore the reasons for the increase in TGF- $\beta$ content, we also tested the OPN content in the supernatant of the co-culture system and found that a hydrogen-rich medium effectively inhibits the secretion of OPN (Fig. 6F). The secreted TGF- $\beta$ cleaves OPN and prevents it from promoting tumor cell growth .

\section{Discussion}

DEN is a carcinogenic drug. In this study, we used DEN to interfere with LX-2 normal liver cells to establish a cell damage model. The experimental results showed that as the concentration of DEN increases, the 
cell activity of LX-2 gradually decreases, and the content of TGF- $\beta$ and TNF- $\alpha$ in the cell supernatant increases. When the concentration of DEN was $200 \mu \mathrm{mol} / \mathrm{L}$, the activity of LX-2 cells was significantly reduced, suggesting that DEN damages the normal liver cells. After different concentrations of DEN were used to induce cell damage, a hydrogen-rich medium was used for intervention, and it was found that the activity of LX-2 cells increased, indicating a protective effect of hydrogen-rich water on the DEN-induced cell damage. In addition, compared to normal LX-2 cells, the expression levels of GP73, TGF- $\beta$, and Smad2 mRNA and protein in DEN-injured LX-2 cells increased but decreased after intervention with hydrogen-rich water. However, the level of Smad2 protein increased continually, indicating that hydrogenrich water protects LX-2 cells from DEN injury by downregulating the expression of GP73 and TGF- $\beta$. Thus, it is inferred that LX-2 cells stimulated by DEN are damaged but have not progressed to the stage of liver cancer, and hence, hydrogen-rich water has no obvious effect on the protein expression of Smad2 in the cells.

In the experiment of DEN on LX-2 cells, TGF- $\beta$ and TNF- $\alpha$ in the supernatant of LX- 2 cells increased slightly, and the expression of GP73,TGF- $\beta$, and Smad 2 mRNA was upregulated, indicating that LX-2 cells appeared to be certain. After the intervention of hydrogen-rich water, the mRNA and protein expression of GP73 and TGF- $\beta$ in the injured LX-2 cells was downregulated, indicating a protective effect of hydrogenrich water. The cells cannot progress to liver cancer cells, and hence, cannot significantly inhibit the expression of Smad2 mRNA and protein. In the co-culture system of liver cancer cells and LX-2, the cell morphology improved after the intervention of hydrogen-rich water; also, inhibition of cell proliferation was observed.

Hydrogen-rich water exerts an inhibitory effect on the proliferation of liver cancer cells. When the number of liver cancer cells reach a certain level, the inhibitory effect of hydrogen-rich water weakens. When liver cancer cells interfere with hydrogen-rich water, hydrogen-rich water inhibits the proliferation of liver cancer cells by significantly inhibiting the upregulation of GP73, TGF- $\beta$, and Smad2 mRNA expression and the upregulation of GP73 protein level. Among these, the increased mRNA and protein expression of TGF- $\beta$ indicates that when the number of liver cancer cells peak, hydrogen-rich water plays a role in the further growth of liver cancer cells via upregulated expression of TGF- $\beta$.

In summary, the inhibitory effect of hydrogen-rich water on HepG2 liver cancer cells and the protection of normal hepatic stellate cells are achieved by regulating the GP73/TGF- $\beta /$ Smad2 signaling pathway, thereby demonstrating the effects of hydrogen-rich water on different cells at various stages. Thus, these findings provide a scientific basis for an in-depth investigation on the role of hydrogen-rich water.

\section{Conclusion}

Hydrogen-rich water has a protective effect on liver cell damage and an inhibitory effect on liver cancer cells produced by regulating the GP73/TGF- $\beta$ signaling path.

\section{Abbreviations}


GP73: Golgi protein 73; TGF - $\beta$ : transforming growth factor; TNF-a: tumor necrosis factor; OPN:

Osteopontin; ELISA: enzyme-linked immunosorbent assay; HRW: hydrogen-rich water; HCC:

Hepatocellular carcinoma; DEN: N-nitrosodiethylamine; HBV: Hepatitis B virus; HCV: Hepatitis C virus; RTPCR: real-time reverse transcriptase polymerase chain reaction; Ct: comparative threshold; SDS-PAGE: sodium dodecyl sulfate polyacrylamide gel electrophoresis; ECL: Emitter Coupled Logic; TBST: Tris Buffered saline Tween.

\section{Declarations}

\section{Acknowledgements}

We would like to thank the researchers and study participants for their contributions.

\section{Authors' contributions}

YL: Protocol/project development; YG: Data collection or management; SJ, YX, YWH: Data analysis; YY, $\mathrm{XBH}$ : Data analysis and manuscript revision; WBC, JWZ, LHC: Manuscript writing/editing. All authors read and approved the fnal manuscript

\section{Funding sources}

Special fund for clinical research of Wu Jieping Medical Foundation

\section{Data availability}

All datasets presented in this study are included in the article/additional fles.

\section{Competing interests}

The authors declare that they have no competing interests.

\section{Consent for publication}

All authors have read and approved the fnal manuscript.

\section{Ethics approval and consent to participate}

Not applicable.

\section{Author details}

${ }^{1}$ Department of interventional medicine, Cancer Hospital Affiliated to Harbin Medical University, Harbin 150081, China.

\section{References}


[1] Liang L, Hong YW. Heterogeneity of liver cancer and personalized therapy. Cancer Letters. 2016; 397 (2):191-197.

[2] Kladney RD, Bulla GA, Guo L, Mason AL, Tollefson AE, Simon DJ, et al. GP73, a novel Golgi-localized protein upregulated by viral infection. Gene. 2000; 249(1): 53-65.

[3] Norton PA, Comunale MA, Krakover J, Rodemich L, Pirog N, D'Amelio A, et al. N-linked glycosylation of the liver cancer biomarker GP73. Journal of cellular biochemistry. 2008; 104(1):136-149.

[4] Kladney RD, Tollefson AE, Wold WSM, Fimmel CJ. Upregulation of the Golgi Protein GP73 by Adenovirus Infection Requires the E1A CtBP Interaction Domain. Virology. 2002; 301(2): 236-246

[5] Malaguarnera G, Giordano M, Paladina I, Berretta M, Cappellani A, Malaguarnera M. Serum markers of hepatocellular carcinoma. Dig Dis Sci. 2010; 55:2744-2755.

[6] Mao Y, Yang H, Xu HF, Lu X, Sang XT, Du SD, et al. Golgi protein 73 (GOLPH2) is a valuable serum marker for hepatocellular carcinoma, Gut. 2010; 59 (12): 1687-1693 .

[7] Tian LY, Wang Y, Xu DB, Gui JH, Jia XW, Tong HL, et al. Serological AFP/Golgi protein 73 could be a new diagnostic parameter of hepatic diseases. Int J Cancer. 2011; 129 (8): 1923-1931.

[8] Zhang YL, Zhang YC, Han W, Li YM, Wang GN, Yuan S, et al. Effect of GP73 silencing on proliferation and apoptosis in hepatocellular cancer. World J Gastroenterol. 2014; 20 (32): 11287-11296.

[9] Morikawa M, Derynck R, Miyazono K. TGF- $\beta$ and the TGF- $\beta$ Family: Context-Dependent Roles in Cell and Tissue Physiology. Cold Spring Harb Perspect Biol. 2016; 8 (5): a021873.

[10] Katsunori Y, Koichi M, Miki M, Takashi Y, Kanehiko S, Kazuichi O. Clinico-Pathological Importance of TGF- $\beta$ /Phospho-Smad Signaling during Human Hepatic Fibrocarcinogenesis. Cancers. 2018; 10(6):183.

[11] Li X, Li L, Liu X, Wu J, Sun X, Li Z, et al. Attenuation of Cardiac Ischaemia-reperfusion Injury by Treatment with Hydrogen-rich Water. Current Medicinal Chemistry. 2019;19 (4): 294-302.

[12] Li L, Liu T, Li X, Liu L, Li S, Li Z, et al. Protein chip and bioinformatic analyses of differentially expressed proteins involved in the effect of hydrogen-rich water on myocardial ischemia-reperfusion injury, International Journal of Medical Sciences. 2019; 16 (9):1254-1259.

\section{Figures}



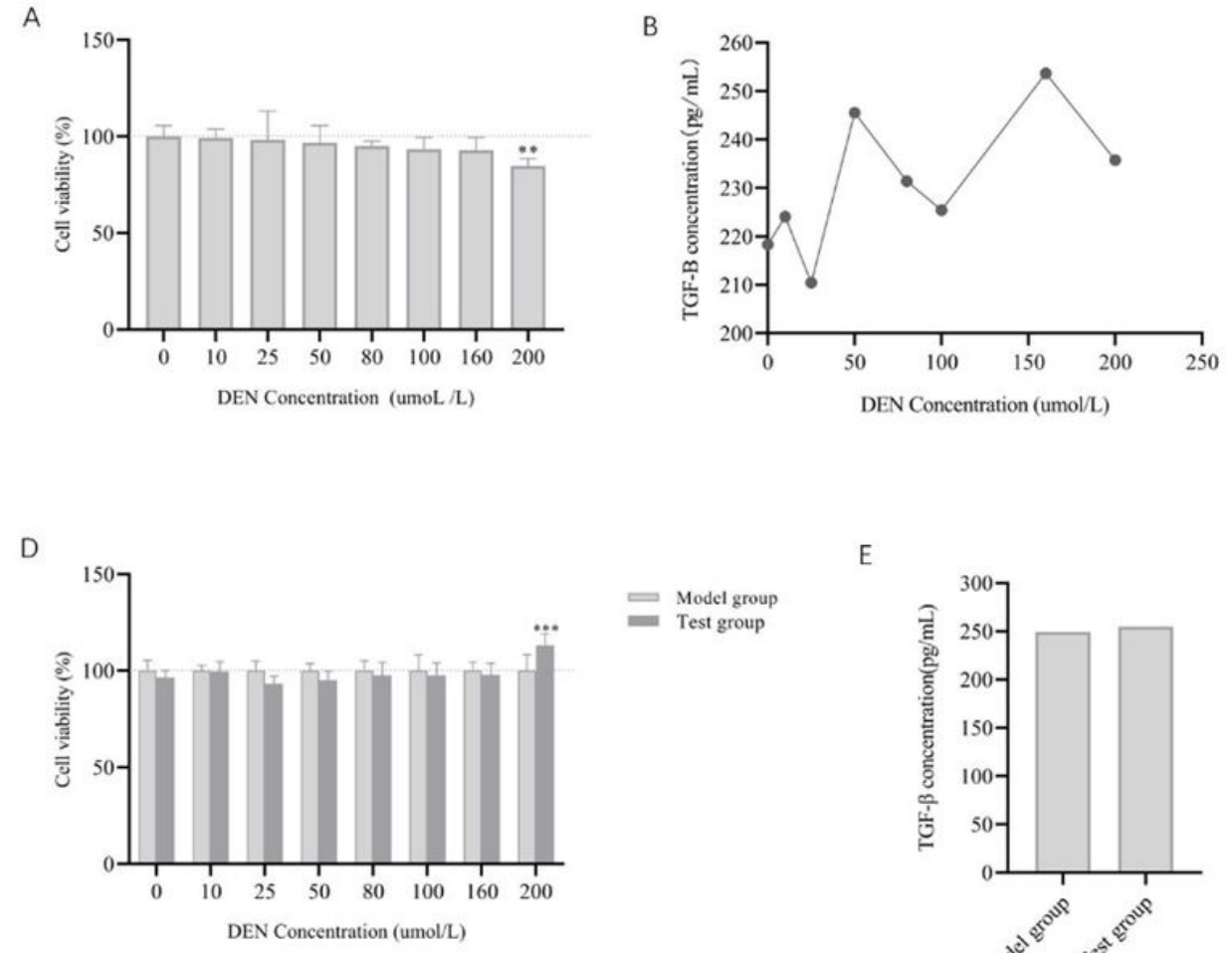

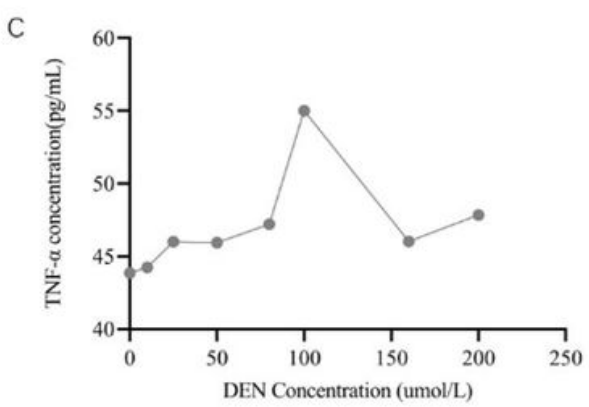

E

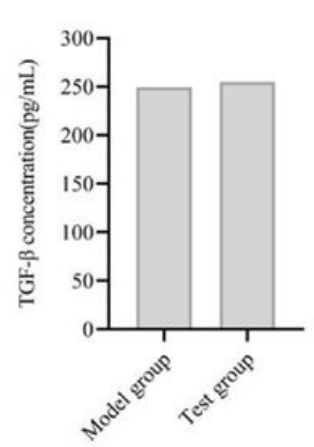

F

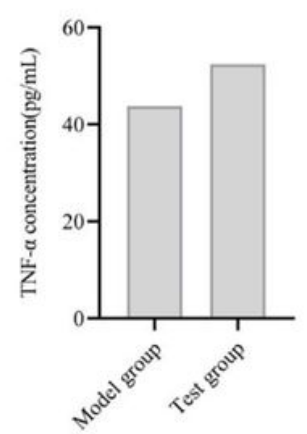

\section{Figure 1}

DEN induces LX-2 cell damage and the effect of hydrogen-rich water intervention. (A) Effect of different concentrations of DEN on LX-2 cell viability. (B) Different concentrations of DEN induce the content of TGF- $\beta$ in the supernatant of LX-2 cells. (C) Different concentrations of DEN induce TNF-a content in the supernatant of LX-2 cells. (D) Hydrogen-rich water intervention with different concentrations of DEN promotes LX-2 cell viability. (E) Hydrogen-rich water intervention with different concentrations of DEN induces TGF- $\beta$ content in LX-2 cells. (F) Hydrogen-rich water interferes with different concentrations of DEN-induced TNF-a in the supernatant of LX-2 cells. 

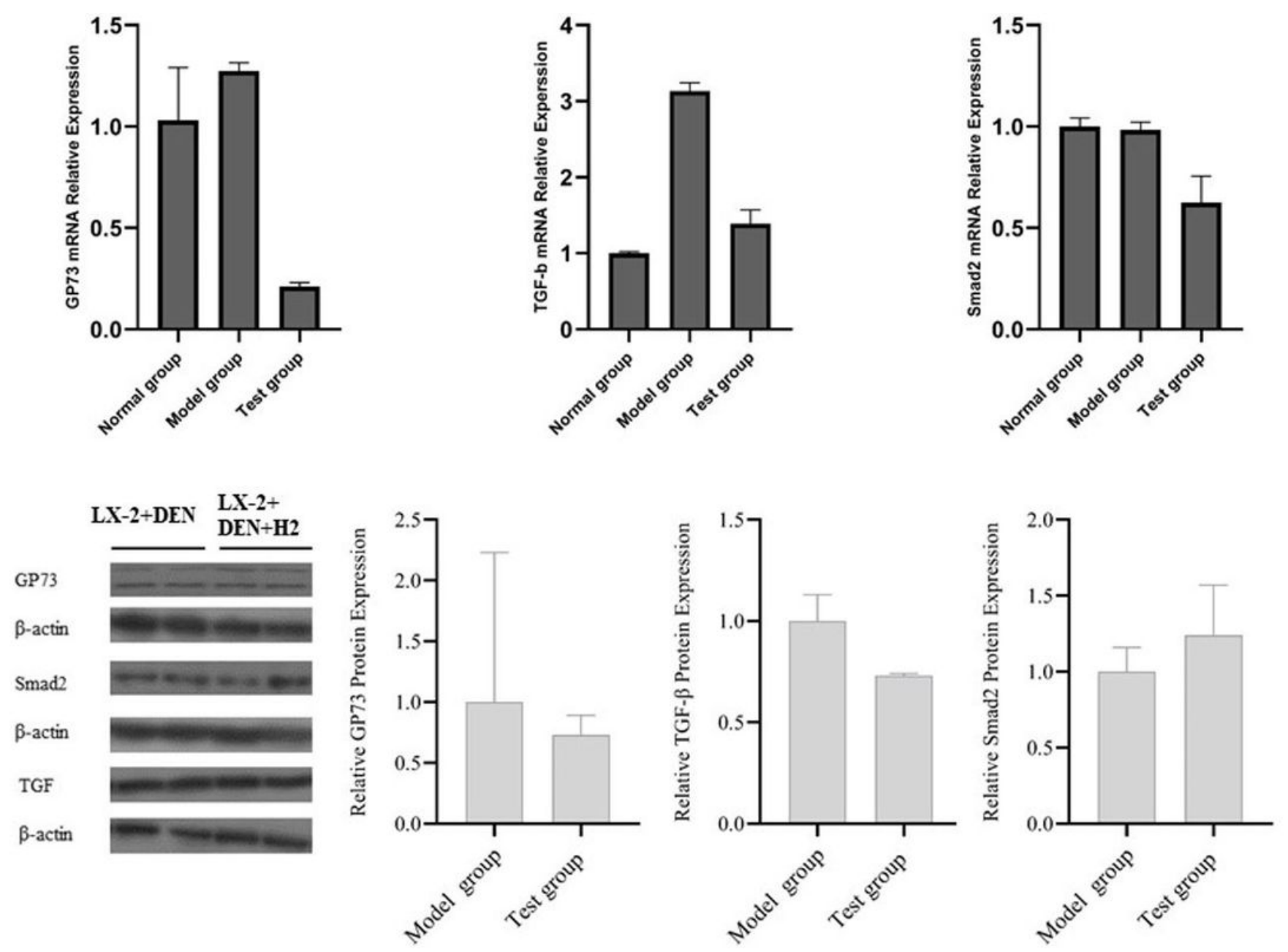

Figure 2

DEN damage and hydrogen-rich water intervention in the changes of key genes and proteins in LX-2 cell pathways. (A) GP73 mRNA in hydrogen-rich water interference in DEN-damaged in LX-2 cells. (B) TGF- $\beta$ mRNA in hydrogen-rich water intervention in DEN-damaged LX-2 cells. (C) Smad2 mRNA in hydrogen-rich water intervention in with DEN-damaged LX-2 cells. (D) Hydrogen-rich water interferes with DEN damage LX-2, as assessed by Western blot. (E) GP73 protein expression in hydrogen-rich water intervention in DEN-damaged in LX-2 cells. (B) TGF- $\beta$ protein expression in hydrogen-rich water intervention in DENdamaged in LX-2 cells. (C) Smad2 protein expression in hydrogen-rich water interference in DENdamaged LX-2 cells. 
A

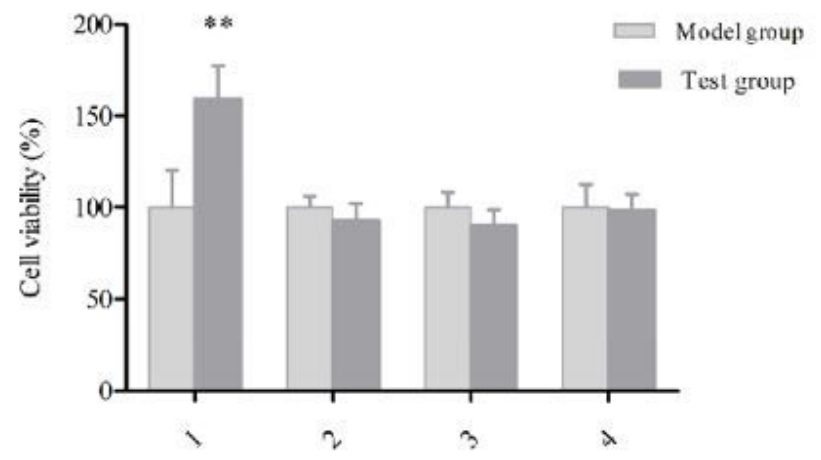

$\mathrm{C}$

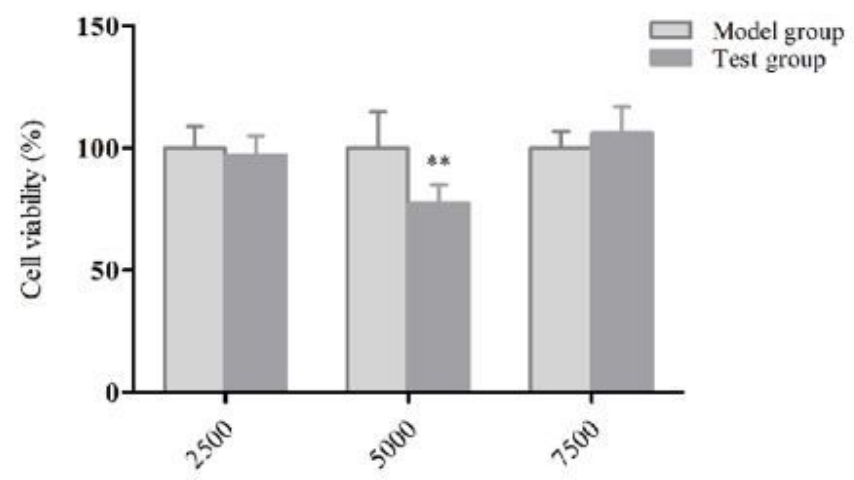

E

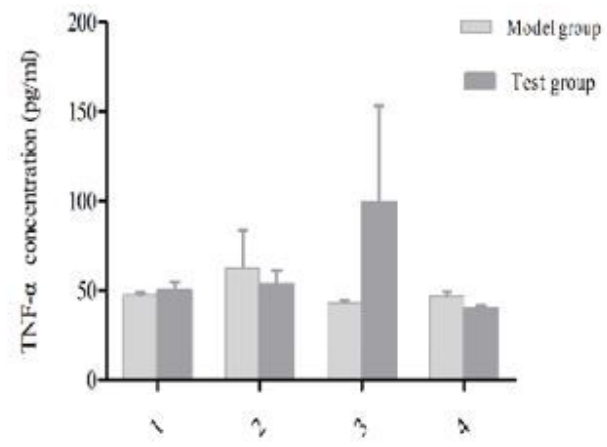

B

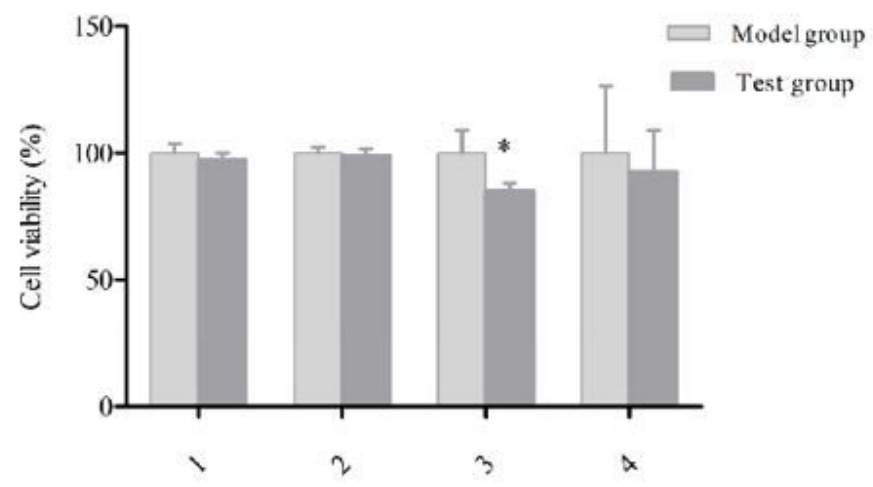

D

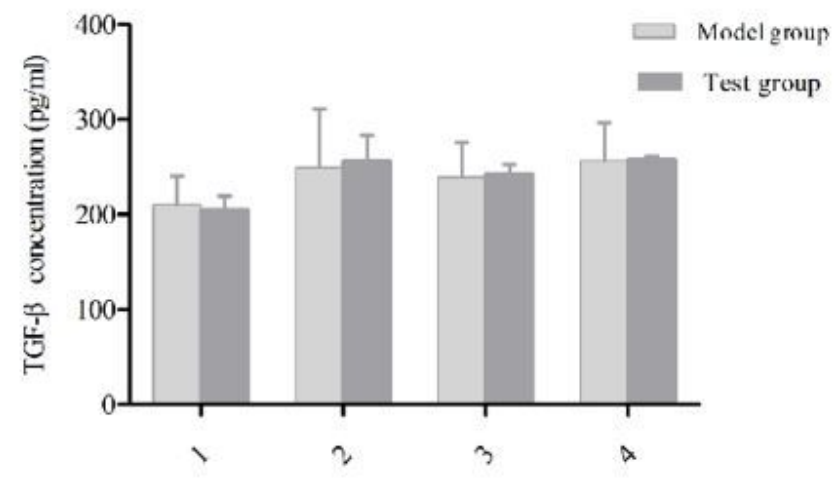

G

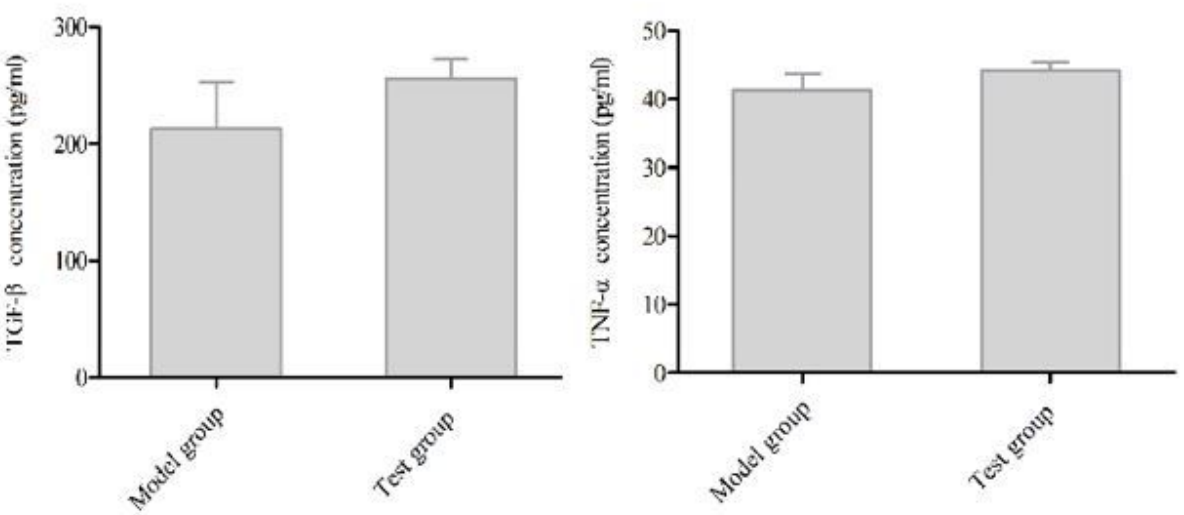

\section{Figure 3}

Study on the effect of hydrogen-rich water on HepG2 cells. (A) Hydrogen-rich water at different times interfered with HepG2 cell viability for $8 \mathrm{~h}$. (B) Hydrogen-rich water at different times interfered with HepG2 cell viability for 24 h. (C) Hydrogen-rich water with different number of HepG2 cell viability. (D) Hydrogen-rich water at different times interferes with TGF- $\beta$ content in HepG2 cell supernatant. (E) Hydrogen-rich water at different times interferes with TNF-a content in HepG2 cell supernatant. $(F)$ Hydrogen-rich water interferes with the content of TGF- $\beta$ in HepG2 supernatant. (G) Hydrogen-rich water interferes with the content of TNF- $\alpha$ in the HepG2 supernatant. 

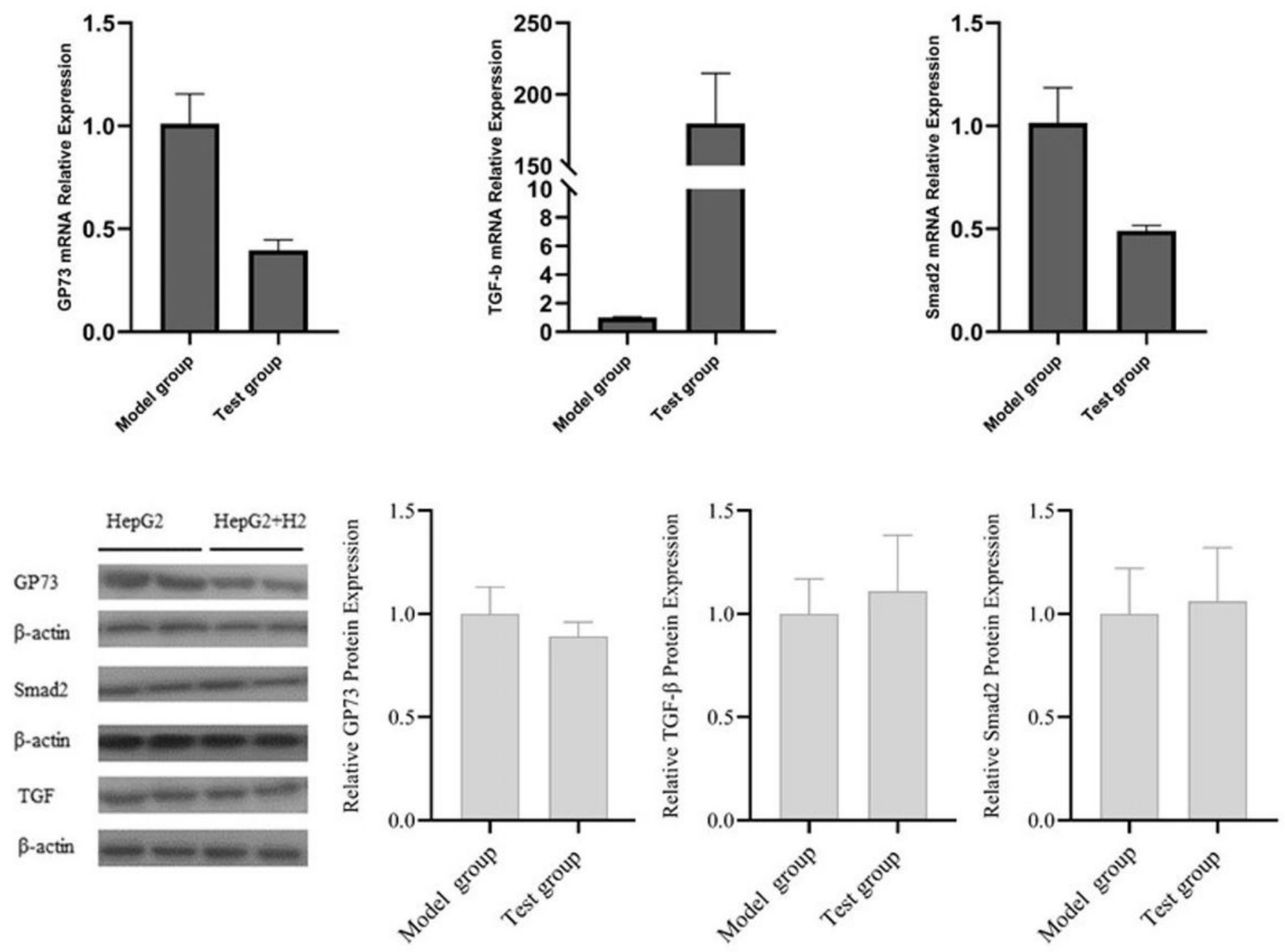

Figure 4

Changes in key genes and proteins in the pathway after hydrogen-rich water intervention in HepG2cells. (A) Hydrogen-rich water interferes with GP73 mRNA in HepG2 cells. (B) Hydrogen-rich water interferes with TGF- $\beta$ mRNA in HepG2 cells. (C) Hydrogen-rich water interferes with Smad2 mRNA in HepG2 cells. (D) Western blot analysis of key proteins in the pathway after intervention with hydrogen-rich water. (E) Hydrogen-rich water interferes with the expression of GP73 protein in HepG2 cells. (F) Hydrogen-rich water interferes with the expression of TGF- $\beta$ protein in HepG2 cells. (G) Hydrogen-rich water interferes with Smad2 protein expression in HepG2 cells. 


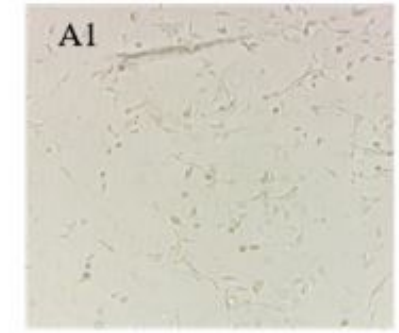

LX-2细胞

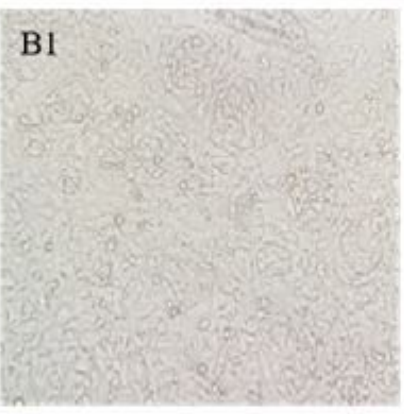

$1: 3$

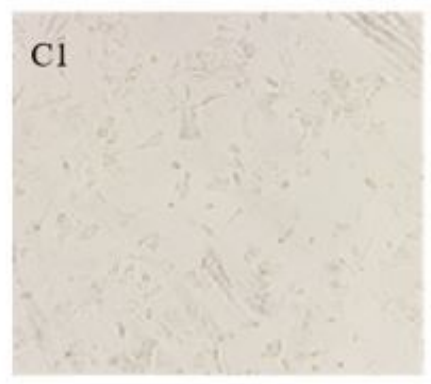

$1: 3$

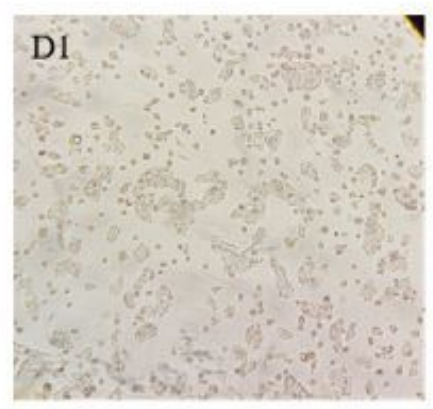

1: 3

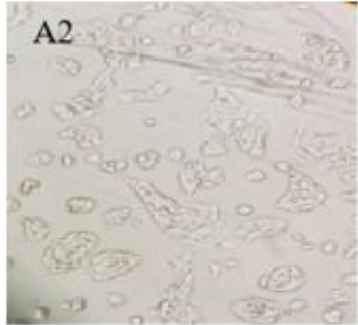

HepG2 (50ul)

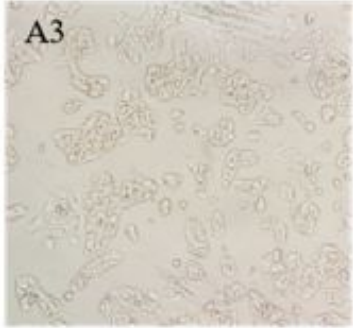

HepG2 (100ul)

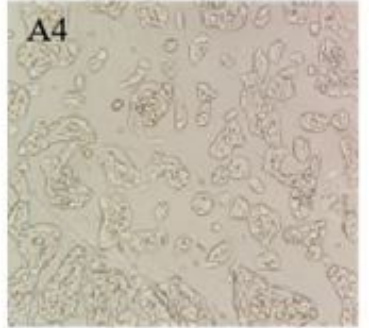

HepG2 (150ul)

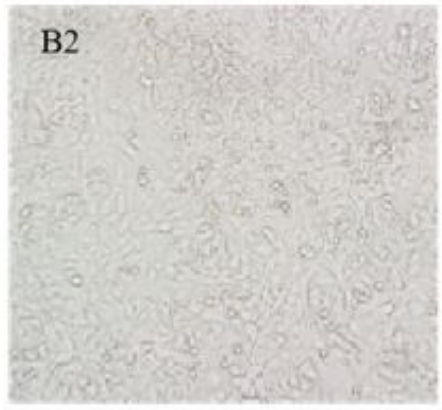

$1: 1$

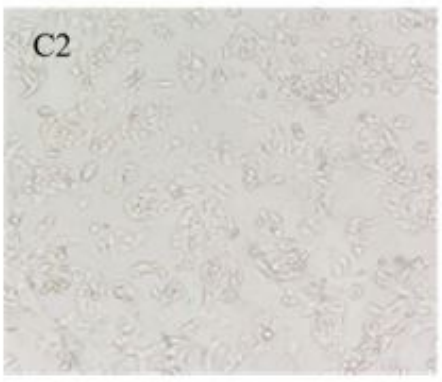

$1: 1$

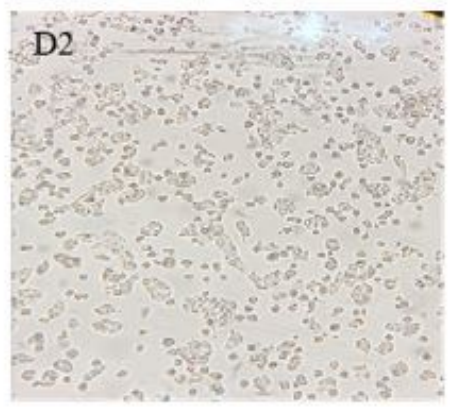

1: 1

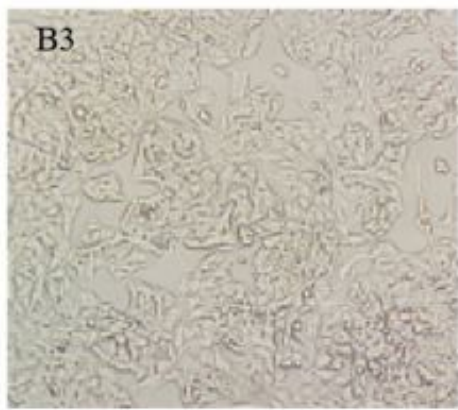

$3: 1$

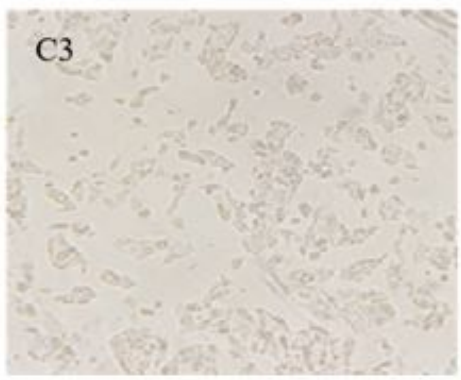

$3: 1$

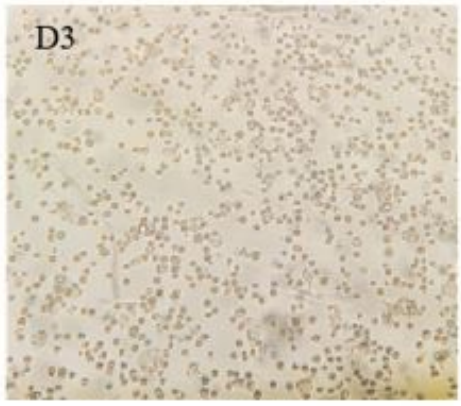

3: 1

\section{Figure 5}

Establishment of co-culture system of LX-2 and HepG2 cells (HepG2:LX-2). (A1) LX-2 cells cultured for $4 \mathrm{~h}$ are evaluated under the microscope. (A2) Density of 2500/well HepG2 was cultured for $4 \mathrm{~h}$. (A3) Density of 5000/wellHepG2 cultured for $4 \mathrm{~h}$ and assessed under the microscope. (A4) Density of 7500/well HepG2 cultured for $4 \mathrm{~h}$ and evaluated under the microscope. (B1-B3) Different ratios of the co-cultivation system for $4 \mathrm{~h}$. (C1-C3) Different ratios in the co-cultivation system for $<10 \mathrm{~h}$ and assessed under the 
microscope. (D1-D3) Different ratios in the co-cultivation system and assessed after $24 \mathrm{~h}$ under the microscope.
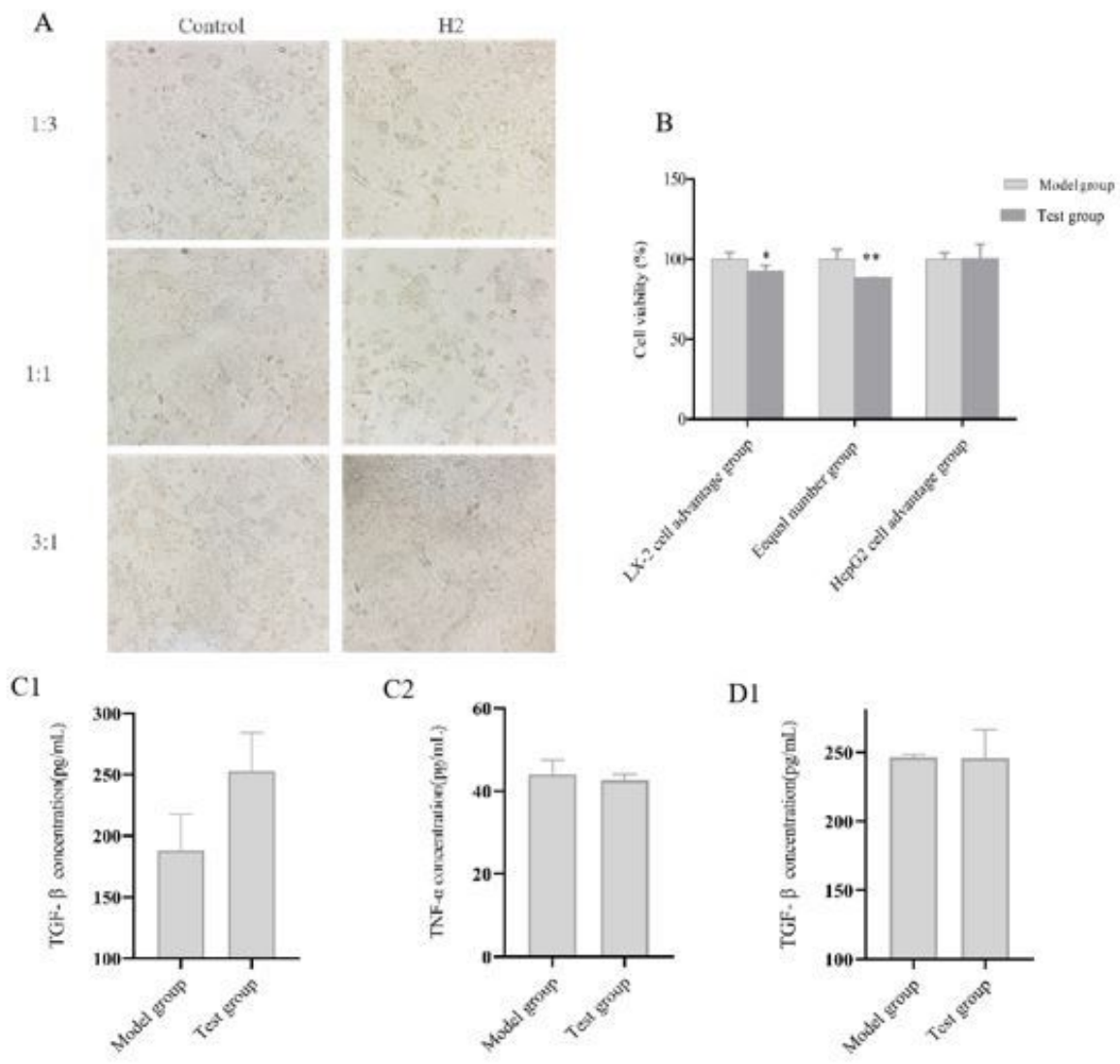

C2

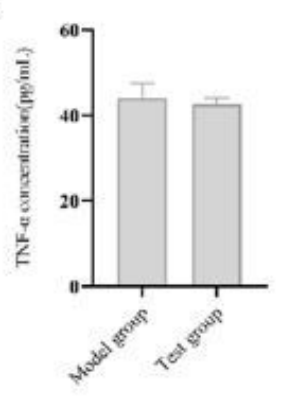

DI

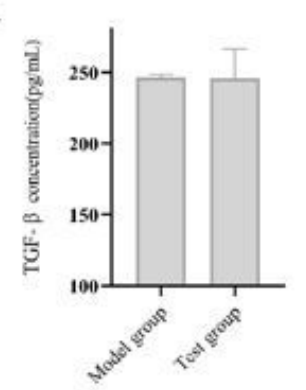

D2

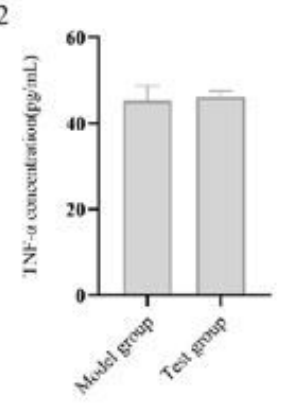

E1

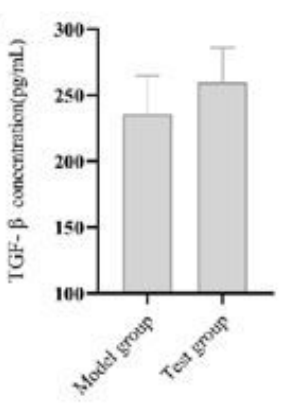

E2

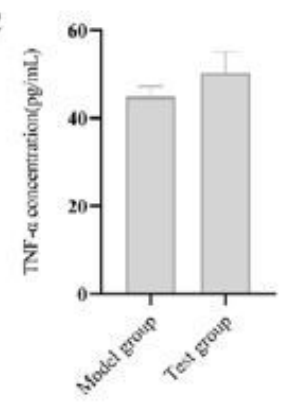

F

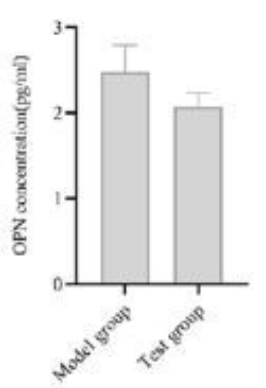

Figure 6

Experimental results of hydrogen-rich water intervention in LX-2 and HepG2 co-culture system (HepG2:LX2). (A) Microscopic picture of the co-culture system after intervention with different ratios of cells and hydrogen-rich water. (B) Effect of hydrogen-rich water intervention on cell viability in different 
ratios. (C1) Hydrogen-rich water increases the content of TGF- $\beta$ in the supernatant of the LX-2 cell dominant growth group. (C2) Hydrogen-rich water decreases the content of TNF-a in the supernatant of the LX-2 cell dominant growth group. (D1) Hydrogen-rich water increases in equal proportion cell growth the content of TGF- $\beta$ in the supernatant of the group. (D2) The content of TNF- $\alpha$ in the supernatant of the cell growth group increased with hydrogen-rich water. (E1) The content of TGF- $\beta$ in the supernatant of the HepG2 cell growth group with the intervention of hydrogen-rich water. (E2) Hydrogen-rich water interferes with the TNF-a content in the supernatant of the HepG2 cell dominant growth group. (F) Hydrogen-rich water interference with the OPN content in the supernatant of the HepG2 cell dominant growth group.

\section{Supplementary Files}

This is a list of supplementary files associated with this preprint. Click to download.

- A.DENLX2Model.xlsx

- B.TGFb2020.6.30.xIsx

- C.TNFa2020.6.30.xIsx

- D.LX22020.6.3.xIsx

- E.TGFb2020.6.30.xIsx

- F.TNFa2020.6.30.xIsx

- Fig2A.xls

- E.TNFa2020.6.30.xIsx

- D.TGFb2020.6.30.xIsx

- F.TGFb2020.6.30.xlsx

- FIG6C1.xlsx

- Fig2C.xls

- Fig4A.xls

- Fig4C.xls

- Fig6B.xlsx

- FIG6C2.xIsx

- FIG6D2.xIsx

- Fig2B.xls

- Fig4B.xls

- FIG6C2.xlsx

- Fig6D1.xlsx

- Fig6E1.xlsx

- FIG6E2.xlsx 
- FIG6F.xIsx

- G.TNFa2020.6.30.xIsx

- grayscalevalue.xlsx

- renamed425d6.xIsx

- renamed36699.xIsx

- renamedad39a.xIsx 